\title{
MOTIVOS DE PARTICIPACIÓN DEPORTIVA Y ORIENTACIÓN DE METAS EN DEPORTISTAS JUVENILES COSTARRICENSES
}

\author{
${ }^{1 y}{ }^{2}$ Garita Azofeifa Elmer $y{ }^{1 y 2}$ Araya Vargas Gerardo \\ ${ }^{1}$ Escuela de Educación Física y Deportes, Universidad de Costa Rica \\ ${ }^{2}$ Escuela Ciencias del Deporte, Universidad Nacional \\ E-mail: garitaazofeifa13@gmail.com
}

\section{Resumen}

Garita-Azofeifa, E. y Araya-Vargas, G. (2007). Motivos de participación deportiva y orientación de metas en deportistas juveniles costarricenses. Revista de Ciencias del Ejercicio y la Salud, 5(1), 24-40. El propósito del estudio fue determinar la importancia de los motivos de participación deportiva y de la orientación de metas según sexo y edad y así mismo, la relación que existe entre los motivos de participación deportiva y la orientación de metas según el sexo en deportistas costarricenses de edad infantil (12 años y menos), juvenil (13 a 19 años) y mayor (20 o más años). Participaron 149 deportistas (68 mujeres, 81 hombres) con edades entre los 8 y los 23 años de edad (15,48 $\pm 3,02$ años de edad) que entrenaban y competían regularmente en deportes como judo, natación, voleibol, baloncesto, atletismo, béisbol, fútbol, tenis de campo, fútsal, taekwondo, gimnasia y triatlón. Se les aplicó el "Cuestionario sobre Motivos para la Participación Deportiva" (Durand, 1988) y el "Cuestionario de Orientación al Ego y a la Tarea" (Balaguer, Castillo y Tomás, 1996). Los resultados indican que no se encontraron diferencias en la importancia que le dan hombres y mujeres a los motivos de participación deportiva y a las orientaciones de metas a la tarea y al ego, sin embargo, se encontró, que para ciertos grupos de edad, algunos motivos son más importantes a la hora de explicar la participación en deporte y también se encontró diferencias para la edad en la orientación de metas a la tarea. El factor forma física, fue el más importante en los tres grupos de edad estudiados, no obstante, en el caso de los sujetos de 12 años o menos, este factor comparte su importancia con el desarrollo de habilidades y los factores sociales, y en los sujetos de 13 a 19 años, forma física comparte importancia solo con desarrollo de habilidades; en los sujetos de 20 años o más, la forma física es claramente el factor motivacional más importante; la diversión y la búsqueda de esparcimiento se destacaron más de los otros factores motivacionales a partir de los 13 años, pero compartiendo importancia con el desarrollo de habilidades. Para los hombres de esta muestra, algunos motivos (búsqueda de esparcimiento y desarrollo de habilidades) se ven influenciados por la orientación de metas a la tarea, otros motivos (autorrealización) pueden ser explicados por la orientación al ego y otros motivos (diversión y liberación de energía) se pueden explicar por ambas orientaciones. Según estos resultados, se contradice parte de los estudios previos. Además, se concluye que la teoría de orientación de metas resultó útil para explicar algunos motivos de participación deportiva de los hombres, pero no tuvo la misma utilidad para explicar los motivos de participación de las mujeres. Estudios futuros deberían profundizar en otros modelos teóricos más integrales, que permitan explicar mejor la motivación de deportistas, especialmente de las mujeres.

PALABRAS CLAVES: Motivos de participación deportiva, orientación de metas, sexo, edad, deporte.

\section{INTRODUCCIÓN}

Una de las áreas que genera mayor interés de investigación dentro de la psicología del deporte, es la referente a los principales motivos por los cuales los niños o los jóvenes se inician en el deporte y las razones por las que sujetos de todas las edades se mantienen realizándolo.

Antes de que Gill, Gross y Huddleston (1983), publicaran el "Participation Motivation Inventory", los instrumentos para medir los motivos de participación eran inespecíficos. Entonces, aparece el auge investigador en el contexto deportivo (Martínez, Andrade y Salguero, 2005), hecho que reconocen Balaguer y Atienza (1994), expresando la importancia de procurar que los entrenadores y monitores deportivos puedan diseñar programas que cumplan con las necesidades de los deportistas contribuyendo con su desarrollo personal.

Para los diferentes investigadores en este campo, los motivos para la participación en deporte son variados y los agrupan en factores llamados de distintas maneras. Por ejemplo, Alderman (1978) presenta una lista de motivos que podrían inducir a los 
deportistas jóvenes a comprometerse en actividades deportivas, entre ellas: necesidad de hacer amistades/intercambio social; oportunidad de influir o controlar a otros; posibilidad de hacer las cosas por sí mismo; realizar actividades caracterizadas por producir tensión, excitación, estrés o depresión; oportunidad de hacer bien algo difícil; probabilidad de recibir refuerzos externos, como aprobación, admiración, trofeos, entre otras y posibilidad de dominar, someter o vencer a otros.

Durand (1988), basado en la traducción del instrumento de Gill y cols. (1983), ubicó 30 motivos de participación deportiva en ocho factores denominados: autorrealización; diversión; liberación de energía; búsqueda de esparcimiento; desarrollo de habilidades; factores sociales; práctica en equipo y forma física.

Por su parte, Castillo, Balaguer y Duda (2000), distribuyeron 10 motivos de práctica deportiva en 3 dimensiones, llamadas: motivos de aprobación social/demostración de capacidad motivos de salud y motivos de afiliación.

Finalmente Goldstein y Krasner (1991) citados por Lorenzo (1997), presentan un listado de cerca de cien motivos por los que los deportistas participan en deporte y los distribuye en 19 categorías, algunas de ellas llamadas: aprobación social, competición, autodominio, estilo de vida, miedo al fracaso, salud y forma física, amistades y relaciones personales, éxito y logros, recompensas tangibles, reconocimiento, entre otras.

Entonces, son muchos los motivos que puede tener un deportista para practicar y aunque es posible identificar los más habituales, éstos varían y son específicos de cada persona, por lo que de las listas expresadas anteriormente, algunos motivos resultan muy familiares y otros ciertamente singulares (Weinberg y Gould, 1996).

Se aprecia entonces lo complejo que resulta el abordaje de este tema, ya que los motivos son diversos e influyen incluso variables como el sexo, la edad de las poblaciones estudiadas, el deporte practicado, las diferencias socioculturales, la satisfacción al practicarlas, el nivel de práctica (principiantes, intermedios, avanzados), entre otros. Sobre cada una de estas y otras variables, se puede hacer referencia extensivamente, sin embargo, se hará mención a la variable sexo y edad del deportista, incluidas en este análisis.

En cuanto a los motivos entre sexos, Gould, Feltz y Weiss (1985) encontraron que las diferencias se deben básicamente a que los hombres participan por estatus social y aunque las oportunidades para las niñas aumentan, son ellos quienes tienen más posibilidad de obtener mejores situaciones deportivas principalmente en el nivel profesional. Otros autores indican que los hombres, le dan mayor importancia al estatus, a la competición, a la liberación de energía (Rosich, 2005; Weinberg y cols. 2000; Longhurst y Spink, 1987; Sapp y Haubenstricker; 1978).

Los resultados de Petherick y Weigand (2002), revelaron que los hombres fueron significativamente más motivados extrínsecamente que las mujeres. Éstas, por su lado, suelen tener metas de control de peso y mejorar su apariencia y han demostrado estar orientadas a lo social, lo que sugiere que dan mayor importancia a la relación de pares y un fuerte énfasis social, a la amistad, la diversión, el trabajo en equipo y a la condición física; Por su parte, las mujeres jóvenes australianas dan más énfasis al aprendizaje de las habilidades (Rosich, 2005; Isogai, Brewer, Cornelius, Etnier y Tokunaga, 2003; Weinberg y cols., 2000; Longhurst y Spink, 1987; Gould y cols., 1985).

González, Tabernero y Márquez (2000), encontraron algunas diferencias no significativas en función del sexo, en la que los niños consideran más importantes que las niñas, los motivos orientados al resultado como la competición, los desafíos, alcanzar estatus, hacer aquello en lo que destacan y satisfacer a padres y amigos.

Varios estudios han determinado diferencias entre sexos en cuanto a la preferencia de los motivos para participar en el deporte, sin embargo, Alderman (1978) no determinó diferencias entre sexos, ya que encontró que la afiliación, la superioridad y la activación fueron los motivos más 
importantes para ambos sexos; así mismo, Gill y cols (1983) y Balaguer y Atienza (1994), indican que ambos sexos coinciden en los motivos principales para participar en deporte, diferenciándose únicamente en el orden de prioridades. Entonces, la bibliografía actual no permite tomar una determinación definitiva sobre si existen diferencias entre los motivos de participación deportiva de los hombres y de las mujeres.

En cuanto a la edad del deportista y los motivos para la práctica de deportes, se indica que la motivación de los niños puede ser condicionada por sus padres y sus entrenadores (Bergerone, Cei y Ceridono, 1985). La participación de los niños en el deporte puede darse por una combinación entre múltiples motivos intrínsecos y extrínsecos, entre los cuales se encuentran ser competente, la amistad, el desarrollo de habilidades y la competición (Kim, Chang y $\mathrm{Gu}, 2003$; Wang y Weise-Bjornstal, 1996), aunque se sugiere que en su mayoría son motivaciones intrínsecas, ya que es algo que presenta reto y es disfrutable, así como también participan por motivos extrínsecos como ganar la aprobación externa (Weigand y Broadhurst, 1998) y gustar al entrenador, motivo que resulta más importante cuando son más pequeños (Williams y Cox, 2003).

Investigando los motivos de participación deportiva de niños tenistas y futbolistas, González y cols. (2000), encontraron que ambos grupos concedieron mayor importancia a los motivos: mejorar el nivel, estar bien físicamente, divertirme, hacer ejercicio, mantenerse en forma, aprender nuevos movimientos o habilidades y hacer nuevos amigos; resultados que están de acuerdo con lo encontrado por Gould y cols. (1985), lo que demuestra que independientemente del deporte practicado, los motivos para practicarlo son similares, dejando claro que los jóvenes se acercan más al deporte por motivos intrínsecos que extrínsecos. Contradiciendo lo anterior, Weinberg y cols. (2000), encontraron que las razones más mencionadas fueron extrínsecas y relacionadas con la competición, así como han mostrado que la afiliación social, el estatus y el reconocimiento son importantes razones extrínsecas por las que los niños y adolescentes participan en deportes. En resumen, hay controversia sobre los principales motivos para la práctica de deportes en infantes.

Estudios epidemiológicos en Estados Unidos y en Canadá indican que con el aumento en la edad, el comportamiento hacia el ejercicio tiende a disminuir, especialmente en los años de la adolescencia (Nigg, 2003). Al llegar la adolescencia disminuye la actividad física en ambos sexos pero principalmente en las mujeres, por el asunto de la pérdida de feminidad y la discriminación asociada a su participación en el deporte (Cale, 1996; Cookburn, 2000).

En cuanto a los jóvenes, entre los principales motivos de participación se menciona la diversión (Campbell, MacAuley, McCrum y Evans, 2001; Weinberg y cols., 2000), aprender nuevas habilidades (Weinberg y cols., 2000), mejorar su forma física (Rosich, 2005), competir (Hellandsig, 1998; Nigg, 2003) y cooperar con otros, aprender a tomar riesgos y a auto-manejarse, la posibilidad del éxito y el fracaso (Nigg, 2003) y obtener estatus, prestigio y reconocimiento (Alderman y Wood, 1976; Williams y Cox, 2003).

Los adultos jóvenes (de edad universitaria) pasan por un periodo crítico en cuanto a la participación deportiva, ya que es aquí donde la gran mayoría de estudiantes de ese nivel abandonan el hábito de hacer ejercicio, pues deben dedicar muchas más horas al estudio y reducir las de ocio (Blasco, Capdevilla, Pintanel, Valiente y Cruz, 1996), además la adherencia al ejercicio disminuye con la edad ya que un tercio de la población de adultos reporta no tener una rutina de ejercicio regular (Smith y Storandt, 1997). Para los adultos, los motivos de participación más importantes son la competición, la capacidad personal, las relaciones sociales, aventura, forma física e imagen personal (Pavón, Moreno, Gutiérrez, y Sicilia, 2003). Por tanto, si se desea un incremento en el nivel de motivación para la práctica de adultos de edad universitaria, se debe hacer hincapié en las habilidades básicas, en la diversión y en las relaciones grupales, no 
tanto en la popularidad, la recompensa externa y la competición (Tabernero, 1998).

Así, respecto a la variable edad, está claro que los motivos de participación deportiva varían en los grupos de deportistas de diferente edad, lo que es importante porque como lo recomiendan Castillo, Balaguer y Duda (2000), analizar los motivos de participación deportiva, le permite a los entrenadores organizar las actividades procurando que los deportistas obtengan lo que buscan, sin embargo, este tipo de estudios no permiten conocer cuál es el proceso motivacional que los acerca al deporte. Por este motivo se hace necesaria la utilización de marcos teóricos que permitan un análisis más profundo de la descripción de estos procesos, teorías que permitan facilitar la adherencia a los programas deportivos y evitar el abandono (Castillo y cols., 2000). Esto confirma lo expresado por Weinberg y cols. (2000), quienes indican que los estudios más recientes y contemporáneos están más frecuentemente basados en la teoría.

Desde el punto de vista socio-cognitivo, las conductas de logro indican que las personas difieren en la manera en que definen el éxito y el fracaso, mientras intentan demostrar que son competentes y evitan demostrar lo contrario. La teoría de las orientaciones de metas (Maehr y Nicholls, 1980; Nicholls, 1984) propone la existencia de dos perspectivas de metas independientes, que han sido nombradas de diferente forma; algunos autores se han referido a éstas como metas de aprendizaje y metas de rendimiento (Dweck, 1986; Dweck y Elliot, 1983; Dweck y Leggett, 1988), otros las llaman, metas de maestría y habilidad (Ames 1992; Ames y Archer, 1988); de maestría y dominio (Roberts y Treasure, 1995), mientras Nicholls (1989) usó los términos de orientación de metas a la tarea y orientación de metas al ego (Valdés, 1996).

Nicholls (1984) debatió que los individuos en situaciones de desempeño operan bajo estas dos diferentes perspectivas de metas, a la tarea y al ego, y dependiendo de la que hayan adoptado prioritariamente, tienen concepciones diferentes de su habilidad.
Para la orientación de metas a la tarea, Cervelló y Santos-Rosa (2001), mencionan que la persona se esfuerza en obtener los logros personales. Por su parte, Seifriz, Duda, y Chi (1992), expresaron que las percepciones de habilidad son de auto referencia, juzga su nivel de habilidad de acuerdo a cuanto ha aprendido o mejorado progresivamente; es conciente de que es necesario un mayor esfuerzo para dominar una destreza y se percibe habilidoso cuando está teniendo éxito en la tarea. Simons, Dewitte y Lens (2003), detallaron que el sujeto con esta orientación se concentra en realizar la destreza hasta dominarla y alcanzar mejoras personales, frecuentemente demuestran alta capacidad, toman retos exigentes, se esfuerzan al máximo, tiene motivación intrínseca y persisten en el tiempo, porque quieren desarrollarse.

Por su parte en la dimensión de orientación al ego, el individuo pone sus metas para demostrar un rendimiento superior a los demás (Cervelló y Santos-Rosa, 2001), por demostrar habilidad a través de la comparación social con otros individuos.

Cuando la implicación al ego prevalece, el nivel de habilidad es construido con la referencia del rendimiento de los otros. Para percibir alta habilidad, es este caso, el deportista debería rendir más que otros en determinada tarea (Seifriz y cols, 1992).

Algunos estudios (Vlachopoulos, Biddle, Fox, 1996; Simons y cols., 2003) mencionando lo expuesto por Nicholls (1989), expresan sobre los sujetos orientado al ego no toman retos, ni se esfuerzan, no tienen motivación intrínseca y tampoco persisten en el tiempo, porque su principal interés no es desarrollarse; por lo general, cuando perciben que su nivel de habilidad es bajo, están a la espera del fracaso ante cualquier situación, porque son incapaces de hacerlo en forma exitosa y se retiran del deporte.

Como lo expresó Duda (1989), los deportistas jóvenes que están principalmente orientados al ego y poco orientados a la tarea, se comportan de diferente manera en la experiencia deportiva, lo que los lleva a no mantenerse por largos periodos de tiempo en 
la práctica del deporte. Este estudio, al igual que el de Treasure y Roberts (1994), mostró que la relación entre tarea y ego se relaciona negativamente con un grupo de variables como percepción de habilidades, satisfacción deportiva y disfrute del deporte.

En cuanto al análisis de las orientaciones de metas según sexo, Zúñiga (2005), en una investigación con mujeres, determinó que la principal orientación motivacional de las participantes fue hacia la tarea, resultados que concuerdan con lo encontrado en la literatura que indica que las mujeres tienden a ser intrínsecamente motivadas y que suelen ser más orientadas a la tarea que los hombres (White, Kavussanu y Guest, 1998; Balaguer, Castillo y Tomás, 1996). Por otro lado, White y Duda (1994), encontraron que los hombres fueron menos orientados a la tarea que las mujeres, resultados que se relacionan con lo expuesto por Battista, (1990); Duda, (1989); Gill y cols. (1983) quienes indican que los hombres se orientan más al ego que las mujeres.

Otros estudios no han podido confirmar las diferencias entre la orientación de metas entre los sexos (Duda y Horn, 1993; White y Zellner, 1996). Por ejemplo, Etnier, Sindman y Hancock (2004), señalan que no encontraron diferencias en cuanto al sexo en orientación de metas al ego, sugiriendo que en la muestra de adultos con que trabajaron existen mujeres que tienen una orientación al ego tan alta como la de los hombres. Igualmente, White y Duda (1994), hallaron que los hombres se mostraron tan altos en la orientación de metas al ego como las mujeres en estudiantes de educación física. Entonces, según estos autores, la tendencia general es que los hombres sean más orientados al ego mientras las mujeres a la tarea, sin embargo, se evidencia que no es clara la relación entre orientaciones de metas en cuanto al sexo, aspecto que amerita más investigación y se puede deber a las diferencias entre las muestras utilizadas.

Por su parte, Papanoionnou y Theodorakis (1994, citados por Zahariadis y Biddle, 2000) encontraron que los deportistas jóvenes orientados al ego se mostraron más interesados en motivos como el reconocimiento y el estatus; contrariamente, los orientados a la tarea están más interesados en el aprendizaje y el dominio personal de las tareas, lo que les hace adoptar más patrones de motivación intrínseca como el desarrollo de habilidades y el disfrute.

En Costa Rica, hay un antecedente previo de investigación, donde se involucran motivos de participación y orientaciones de metas. Zúñiga (2005), en un estudio sobre la motivación para la práctica de actividades acuáticas en mujeres mayores de 30 años, incluyó descriptivamente las variables en cuestión: motivos de participación deportiva (por medio del cuestionario de motivos de participación deportiva o CMPD de Durand, 1998) y la teoría de orientación de metas (por medio del Task and Ego Orientation in Sports Questionnaire o TEOSQ de Duda, Chi, Newton, Walling, Catley, 1995). Sin embargo no correlacionó a estas variables y además, no existió la variable de sexo (todas eran mujeres), ni edad y los sujetos tampoco eran deportistas.

Zahariadis y Biddle (2000), utilizando la versión original del CMPD, llamado Participation Motivation Questionnaire (PMQ; Gill y cols., 1983), analizaron, en escolares ingleses, la relación entre la orientación de metas y los motivos de participación en educación física y deportes, en la que encontraron que la orientación a la tarea se relacionó positivamente con los motivos de desarrollo de habilidades y la atmósfera o trabajo de equipo $y$ negativamente con los motivos de reconocimiento y estatus. Por otro lado, la orientación al ego se relacionó alta y positivamente con los motivos de reconocimiento y estatus y negativamente con los motivos de atmósfera de equipo. Sin embargo, no se realizó comparaciones según edad y sexo y su muestra no era de deportistas.

En otro estudio al respecto, Castillo, Balaguer y Duda (2000), analizaron las relaciones entre sexos en las orientaciones de metas y los motivos de práctica deportiva distribuidos en 3 dimensiones, llamadas: motivos de aprobación social/demostración de capacidad, motivos de salud y motivos de 
afiliación. Se determinó que la orientación al ego estaba positiva y significativamente relacionada con los motivos de aprobación social/demostración de capacidad, en ambos sexos. Por otra parte la orientación a la tarea estaba relacionada positivamente con los motivos de afiliación en ambos sexos. En el grupo de los hombres, la orientación a la tarea también apareció positiva y significativamente relacionada con los motivos de salud $(\mathrm{r}=0,37 ; \mathrm{p}<0,01)$. Sin embargo, los autores no se interesaron por diferenciar entre hombres y mujeres ni en cuanto a la edad y su muestra no era de deportistas.

Tal y como se ha evidenciado, dentro de la revisión de literatura realizada (en diferentes revistas y bases de datos de artículos científicos nacionales e internacionales) con el fin de obtener información para el presente documento, la teoría de orientación de metas ha sido analizada a la luz de diferentes variables psicológicas, sin embargo, respecto a los motivos de participación en el deporte, existe controversia. Falta dilucidar más, tanto en la teoría de orientación de metas como en los motivos de participación deportiva, si existen diferencias ligadas al sexo, en distintas edades $y$ en muestras específicas de deportistas. Así mismo, falta conocer más a fondo cómo es que se podrían relacionar los motivos de participación deportiva con la orientación de metas. Se ha encontrado pocos estudios que analicen ambos grupos de variables. En este sentido, la teoría de orientación de metas implica que la orientación al ego y a la tarea influencian la motivación para el deporte (Duda, 1993). White y Duda (1994), han encontrado que los individuos orientados al ego es más probable que reporten motivos asociados con la competición y el reconocimiento/estatus, mientras que los orientados a la tarea darían énfasis al desarrollo de las habilidades, la condición física y en menor grado a la afiliación, formar parte de un equipo y la competición como razones para participar del deporte. Estos resultados deben confirmarse en función del sexo y la edad y en sujetos deportistas.

\section{Justificación:}

La variedad de muestras analizadas en los estudios previos, ha demostrado que puede haber diferencias en las variables de orientación de metas y los motivos de participación deportiva según la población. Gran parte de los estudios previos, fueron realizados, principalmente, con estudiantes y no con deportistas como tales (White y Duda, 1994; Papanoionnou y Theodorakis, 1994; Zahariadis y Biddle, 2000 y Castillo, Balaguer y Duda, 2000). El presente estudio extiende el área, al realizarse en el ambiente deportivo específicamente, analizando el efecto del sexo sobre ambas variables en conjunto.

Las relaciones entre los motivos de participación deportiva y las orientaciones de metas permiten confirmar la teoría de que las personas orientadas a la tarea o al ego, prefieren tales $\mathrm{o}$ cuales motivos de participación. La orientación a la tarea tiende a relacionarse con la creencia de que para tener éxito en el deporte hay que esforzarse, colaborar y cooperar con los compañeros. Por el contrario, la orientación al ego se ha relacionado con la creencia de que el éxito en el deporte se consigue si se tiene una gran capacidad o competencia y mediante el uso de trampas o engaños (Duda, Fox, Biddle y Armstrong, 1992; Guivernau y Duda, 1994). Es de esperarse que a la hora de relacionar las variables de orientación de metas y los motivos de participación deportiva, exista relación entre motivos similares a los mencionados con dichas orientaciones de metas.

Dado que podría existir elementos socioculturales que afecten su apreciación, sería interesante ver cómo se comporta este fenómeno en otro contexto. Por ejemplo, geográficamente, de los estudios revisados, varios han sido realizados con muestras de sujetos europeos (Castillo y cols., 2000; Zahariadis y Biddle, 2000; Papanoionnou y Theodorakis, 1994) y otros en Norteamérica (White y Duda, 1994), por lo que, el presente estudio aporta al conocer si se pueden generalizar los resultados encontrados en 
otras latitudes a los países latinoamericanos. Específicamente, en Costa Rica, sería un estudio pionero al relacionar ambas variables.

No existe claridad en cuanto a la diferencia entre sexos, con respecto a los motivos de participación deportiva y la orientación de metas predominante y además las relaciones que tienen estas variables entre sí. De los estudios previos, solamente el estudio de Castillo y cols (2000) analizó las diferencias entre sexos en las relaciones entre motivos de participación deportiva y las orientaciones de metas. Así mismo, las diferencias en la importancia de los factores motivacionales, según la edad, debe analizarse más, según algunos autores (Weinberg y Gould, 1996).

\section{METODOLOGÍA}

Sujetos: participaron 149 deportistas (68 mujeres, 81 hombres) con edades entre los 8 y los 23 años de edad $(15,48 \pm 3,02$ años de edad). La muestra practicaba disciplinas deportivas como judo (32), natación (28), voleibol (21), baloncesto (18), atletismo (16), béisbol (14), fútbol (10), tenis de campo (4), fútsal (3), taekwondo (1), gimnasia (1) y triatlón (1). La distribución por grupos de edad quedó así: 12 años y menores (10 mujeres, 12 hombres); de 13 a 19 años (51 mujeres, 57 hombres) y mayores de 20 años (7 mujeres, 12 hombres). Esta distribución obedece a un intento por generalizar la clasificación de edades de cada deporte, según la organización de las categorías competitivas por edad (niños y niñas o sea deporte escolar; adolescentes, o sea deporte colegial; deporte mayor y universitario) en el país.

Instrumentos: se utilizó la versión al español del "Participation Motivation Inventory" de Gill y cols., (1983) llamado "Cuestionario sobre Motivos para la Participación Deportiva" (CMPD) por Durand (1988), en el cual los participantes completaron la afirmación "Participo en deporte porque:" seleccionando la importancia que le daban a cada uno de los 30 motivos para participar en deporte que lo componen, por medio de una escala de Likert de 3 puntos, siendo 1: muy importante, 2: algo importante y 3: nada importante. Es de notar la naturaleza inversa de la escala, por lo que a mayor puntaje, menor importancia al motivo correspondiente. Los motivos se agrupan en 8 factores.

La validez factorial del "Cuestionario sobre Motivos para la Participación Deportiva", fue investigada mediante un análisis de componentes principales con rotación varimax por Durand (1988). La consistencia interna, se estableció mediante el cálculo de la media de la correlación entre ítems de cada factor y el estadístico alfa de Cronbach, donde el valor del coeficiente alfa global fue de 0.84, indicando que en su conjunto los ítems de la escala están razonablemente relacionados (Durand, 1988).

Tomando la sugerencia de Balaguer y Atienza (1994), y de González, Tabernero y Márquez (2000), el ítem "Quiero entretenerme" de la versión de Durand (1988), fue cambiado por "Me gusta divertirme" que refleja mejor el valor concedido en la versión original de Gill y cols., (1983). Para efectos de este estudio, se obtuvo el puntaje promedio de respuesta de cada factor del CMPD, con el fin de poder comparar, por sexo y grupo de edad, la importancia de cada factor.

Para analizar la orientación de metas se utilizó la versión al español del TEOSQ "Task and Ego Orientation in Sport Questionnaire" (Duda, Chi, Newton, Walling y Cattley, 1995) llamado "Cuestionario de Orientación al Ego y a la Tarea" (Balaguer, Castillo y Tomás, 1996). El TEOSQ consta de 13 ítems, 6 pertenecientes a la orientación al ego y 7 a la orientación a la tarea. En las instrucciones se pide a los sujetos que completen la afirmación: "Yo me siento más exitoso en el deporte cuando...". Las respuestas se recogen mediante una escala de puntuación de tipo Likert del uno al cinco (muy en desacuerdo $=1$, en desacuerdo $=2$, neutral o indiferente $=3$, de acuerdo $=4$, y muy de acuerdo $=5$ ).

En cuanto al análisis de las propiedades psicométricas de la versión al español del TEOSQ, (Balaguer y cols., 1996) analizaron 
la fiabilidad de ambos factores en forma independiente. El coeficiente alpha alcanzó valores adecuados en ambos factores siendo menor el obtenido en el factor "Orientación a la tarea" $(0,78)$, mientras el de "Orientación al ego" fue de 0,80 .

Una versión similar de este instrumento ha sido utilizada en sujetos costarricenses en dos estudios, por Araya (2000) (futbolistas de primera y segunda división) y por Villegas (2003) (en niños futbolistas).

Procedimiento: primeramente se buscó evitar momentos en los que los deportistas se vieran inmersos en estrés pre y post competencia, por lo que fueron localizados minutos antes a sus sesiones de entrenamiento o cuando eran espectadores de competencias deportivas. En algunos casos, fue necesario solicitar la autorización de sus entrenadores o miembros del cuerpo técnico. Las muestras más importantes fueron obtenidas durante el Campeonato Iberoamericano de Judo 2005 con sede en el Gimnasio Nacional de la Sabana; los Juegos Nacionales ICODER 2005 realizados en Cartago y el torneo infantil de béisbol en el Parque Antonio Escarré. Antes de la administración de los cuestionarios, todos los deportistas recibieron instrucciones sobre la importancia de su sinceridad en la respuesta, recalcándose el hecho de ser anónimo, por lo que podían expresarse abiertamente sobre cada uno de los ítems. Fueron instruidos grupalmente sobre los objetivos y las principales indicaciones para formar parte del estudio, para el cual participaron voluntariamente y se les indicó que cualquier duda o situación que no estuviera completamente clara con los ítems de los cuestionarios la evacuaran con el investigador presente. Los cuestionarios fueron aplicados en forma individual por cada uno de los deportistas, con una duración aproximada de 10 minutos. Una vez recolectados los cuestionarios, se procedió a realizar los análisis.

Análisis estadístico: como estadística descriptiva se aplicaron promedios y desviaciones estándar. Como estadística inferencial se aplicaron análisis de varianza de dos vías (sexo y grupo de edad) para cada orientación de metas y análisis de varianza de tres vías (sexo, grupo de edad y factor motivacional) para los puntajes promedio de los factores motivacionales del CMPD. También se aplicaron correlaciones de Pearson, para relacionar las orientaciones de metas con los factores motivacionales. Como post hoc, en los análisis de varianza (ANOVA), se aplicó la prueba de Bonferroni y el LSD (Least significant difference) y el análisis de efectos simples, según fuese necesario. Los análisis se realizaron con el programa estadístico SPSS (Statistical Package for the Social Sciences) versión 8.0 para Windows.

\section{RESULTADOS}

A continuación se presenta la tabla 1 en la que se expresan los resultados obtenidos con la aplicación de los ANOVA, indicándose el nivel de significancia en los casos en que resultó significativo y (n.s) en los que no lo fue.

Tabla 1. Valores de F de ANOVA de tres vías (edad, sexo y factor motivacional) y de dos vías (edad y sexo) aplicados en los deportistas de la muestra

\begin{tabular}{|c|c|c|c|c|c|c|c|}
\hline & F edad & F sexo & F edad X sexo & F factor & F factor $\mathrm{X}$ edad & F factor X sexo & F factor X edad X sexo \\
\hline $\begin{array}{l}\text { MOTIVOS DE PARTICIPACIÓN DEPORTIVA } \\
\text { Importancia promedio }\end{array}$ & 2,701 (n.s) & 0,485 (n.s) & 0,071 (n.s) & $17,798 *$ & $4,554 *$ & 0,938 (n.s) & 1,341 (n.s) \\
\hline ORIENTACIÓN DE METAS & & & & & & & \\
\hline $\begin{array}{l}\text { Orientación de metas a la tarea } \\
\text { Orientación de metas al ego }\end{array}$ & $\begin{array}{c}5,248^{* *} \\
0,02(\mathrm{n} . \mathrm{s})\end{array}$ & $\begin{array}{l}0,139 \text { (n.s) } \\
1,089 \text { (n.s) }\end{array}$ & $\begin{array}{l}0,678(\mathrm{n} . \mathrm{s}) \\
1,812(\mathrm{~ns})\end{array}$ & ------ & ------ & ------ & ------ \\
\hline
\end{tabular}

Nota: * significativos al nivel $\mathrm{p}<0,01$ 
Según la tabla 1 se encontró interacción significativa entre el factor motivacional y el grupo de edad. El análisis de efectos simples mostró que los factores en los que existieron diferencias según el grupo de edad fueron el factor diversión, búsqueda de esparcimiento y forma física. Así mismo, con respecto a las orientaciones motivacionales, se observa que solo existieron diferencias significativas para la orientación a la tarea según grupo de edad. Es decir que, tanto para los factores motivacionales como para las orientaciones de metas no se encontró diferencias según el sexo. En el gráfico 1, se ilustra la interacción encontrada para los puntajes promedio de importancia de los factores motivacionales.

Gráfico 1. Interacción entre grupo de edad y factor motivacional en hombres y mujeres deportistas costarricenses

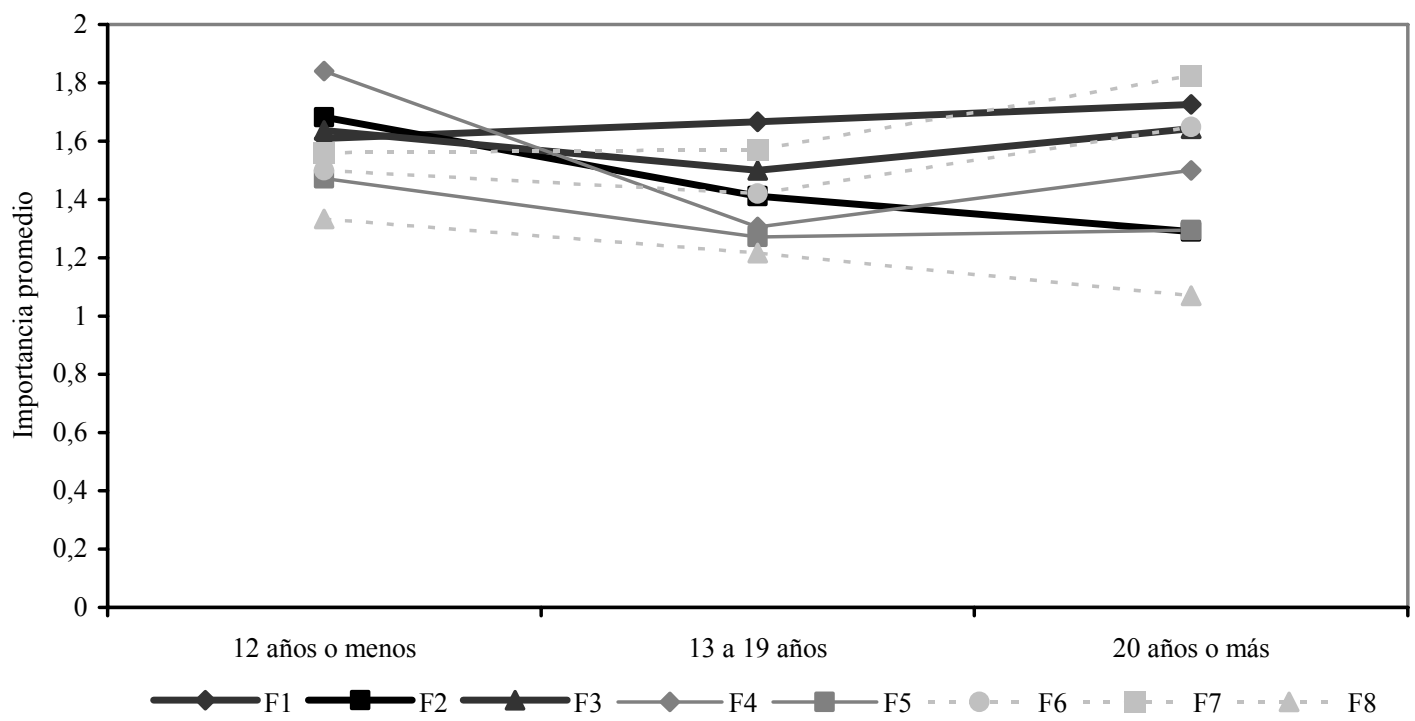

El análisis de efectos simples mostró que, para el factor diversión, hubo diferencias significativas entre los grupos de edad. La tendencia observada es que a mayor edad de los sujetos, mayor importancia a este factor, ya que en el grupo de 12 años, estos motivos fueron menos importantes significativamente que para los otros dos grupos (13-19 años y grupo de 20 años; promedios de $1,6818 \pm 0,43 ; 1,412 \pm 0,393$ y $1,2895 \pm 0,279$ respectivamente; ver tabla 1 y gráfico 1). Se debe aclarar que, al aplicarse una escala de puntuación inversa en el CMPD, un puntaje alto representa menor importancia para el grupo de deportistas.

$$
\text { Para el factor búsqueda de }
$$

esparcimiento, se da el mismo fenómeno, siendo éste menos importante significativamente para los deportistas de 12 años o menos, en comparación con los grupos de edad de 13-19 años y los de 20 años o más (los promedios obtenidos fueron: $1,8409 \pm 0,624 ; \quad 1,3056 \pm 0,403 \quad$ y $1,5 \pm 0,5$ respectivamente; ver tabla 1 y gráfico 1 ).

Finalmente, en cuanto al factor forma física, las diferencias se encontraron entre el grupo de 12 años o menos y el grupo de 20 años o más, siendo ese factor más importante para estos últimos. Los promedios obtenidos, fueron respectivamente: $1,3333 \pm 0,356 ; 1,216 \pm 0,278$ y $1,0702 \pm 0,178$ (ver tabla 1 y gráfico 1). Hasta aquí, los resultados indican que, la tendencia respecto a la importancia dada a los factores diversión, 
búsqueda de esparcimiento y forma física, se acrecienta con la edad, en los deportistas analizados.

En cuanto a la importancia de cada factor dentro de cada uno de los grupos de edad, el análisis de efectos simples mostró que el factor motivacional forma física, fue significativamente el más importante en los tres grupos de edad. En los sujetos de 12 años o menos, la importancia de ese factor fue compartida por los factores desarrollo de habilidades y factores sociales (no hubo diferencias entre estos y forma física), no obstante estos dos últimos factores no se distinguieron tanto de los demás, como fue el caso de la forma física (factores sociales solo fue superior en importancia a la búsqueda de esparcimiento; el desarrollo de habilidades fue más importante que diversión, liberación de energía y búsqueda de esparcimiento); en general en este grupo, los factores búsqueda de esparcimiento, liberación de energía, diversión y autorrealización fueron los menos importantes. En el grupo de 13 a 19 años, la forma física fue más importante que todos los demás factores, salvo por el desarrollo de habilidades con quien no difirió; aquí se destaca con mayor claridad a factores poco importantes como la autorrealización (menos importante que todos salvo por el factor trabajo en equipo con quien no difiere) y el trabajo en equipo; en este grupo se observa un comportamiento diferente al de los sujetos menores de 12 años con respecto a la importancia de la diversión (más importante que la autorrealización, la liberación de energía y que el trabajo en equipo, pero menos importante que la búsqueda de esparcimiento, el desarrollo de habilidades y la forma física) y de la búsqueda de esparcimiento (más importante que autorrealización, diversión, liberación de energía, factores sociales y trabajo en equipo y menos importante que la forma física). En el grupo de 20 años o más, se encontró que la forma física fue más importante que todos los demás factores motivacionales, sin embargo, la diversión y la búsqueda de esparcimiento (aunque ambos fueron menos importantes que la forma física) fueron factores motivacionales importantes en comparación con los demás (salvo por la búsqueda de esparcimiento con el cual no difieren).

Con respecto al análisis de la orientación de metas en cuanto a los grupos de edad y el sexo, solamente se encontró diferencias significativas para la orientación de metas a la tarea en los grupos de edad y no en el sexo. Los promedios obtenidos indican que a mayor edad, mayor es la importancia que dan los deportistas a la orientación de metas a la tarea $(21,82 \pm 7,82$ para el grupo de 12 años o menos; $24,69 \pm 4,57$ para el grupo de 13-19 años y de $26,84 \pm 2,5$ para los de 20 años o más).

En la siguiente tabla se observa el valor del coeficiente de correlación de Pearson, el nivel de significancia y el porcentaje de varianza explicada $\left(r^{2} \times 100\right)$ cuando se correlacionaron los factores de motivos de participación deportiva (componentes del CMPD) y las orientaciones a la tarea y al ego del TEOSQ, en los 81 hombres y 68 mujeres de la muestra.

Tabla 2. Resumen de correlaciones de Pearson y porcentajes de varianza compartida

\begin{tabular}{|c|c|c|c|c|}
\hline \multirow[t]{2}{*}{ NOMBRE DEL FACTOR } & \multicolumn{2}{|c|}{ ORIENTACIÓN DE METAS A LA TAREA } & \multicolumn{2}{|c|}{ ORIENTACIÓN DE METAS AL EGO } \\
\hline & HOMBRES & MUJERES & HOMBRES & MUJERES \\
\hline Autorrealización & n.s. & n.s. & $0,318^{*} ; 10,11 \%$ & $0,252 * ; 6,35 \%$ \\
\hline Diversión & $0,354 * * ; 12,5 \%$ & n.s. & $0,305 * ; 9,3 \%$ & n.s. \\
\hline Liberación de energía & $0,220 * ; 4,84 \%$ & n.s. & $0,277 * ; 7,67 \%$ & n.s. \\
\hline Búsqueda de esparcimiento & $0,224 * ; 5,01 \%$ & n.s. & n.s. & n.s. \\
\hline Desarrollo de Habilidades & $0,346^{*} ; 11,97 \%$ & n.s. & n.s. & n.s. \\
\hline Factores Sociales & n.s. & n.s. & n.s. & n.s. \\
\hline Trabajo en equipo & n.s. & n.s. & n.s. & n.s. \\
\hline Forma Física & n.s. & n.s. & n.s. & n.s. \\
\hline
\end{tabular}

Nota: Los valores de $\mathrm{r}$ originales presentan signos negativos debido a que se utiliza una escala (CMPD) cuya naturaleza es inversa ( $1=$ muy importante; $2=$ algo importante; $3=$ nada importante). Para efecto de evitar interpretaciones incorrectas se presentan los valores de las correlaciones invertidos. $* *$ significativos al nivel $\mathrm{p}<0,01 ; *$ significativos al nivel $\mathrm{p}<0,05$ 
Como se puede apreciar en la tabla anterior, para la muestra de hombres $(n=81)$ varios factores correlacionaron positivamente con la orientación de metas a la tarea (diversión, liberación de energía, búsqueda de esparcimiento y desarrollo de habilidades). Por su parte, con la orientación de metas al ego correlacionaron positivamente los factores autorrealización, diversión y liberación de energía. En las mujeres participantes $(n=68)$ únicamente un factor correlacionó significativamente con alguna de las orientaciones $y$ fue el factor autorrealización con la orientación de metas al ego. Además, se encontró una correlación positiva y significativa entre la orientación de metas a la tarea y la orientación de metas al ego en los hombres de la muestra $\left(r=0,368^{*}\right.$; $\mathrm{p}<0,01)$ representando un porcentaje de varianza compartida entre ambas variables de $13,54 \%$. Por el contrario, al correlacionar la orientación de metas a la tarea y al ego en las mujeres, la correlación no fue significativa $(\mathrm{r}=0,044 ; \mathrm{p}=0,721)$ con un porcentaje de varianza compartida entre ambas variables de $0,19 \%$. Para aclarar las relaciones significativas encontradas en los hombres, respecto a los motivos de participación deportiva y las orientaciones de metas, se presenta el esquema 1, en el que se aprecian los valores porcentuales de la varianza explicada en común entre pares de variables.

Esquema 1. Porcentajes de varianza explicada $\left(\mathrm{r}^{2 * 100}\right)$ entre motivos de participación deportiva y orientación de metas en hombres (corresponden a correlaciones significativas encontradas)

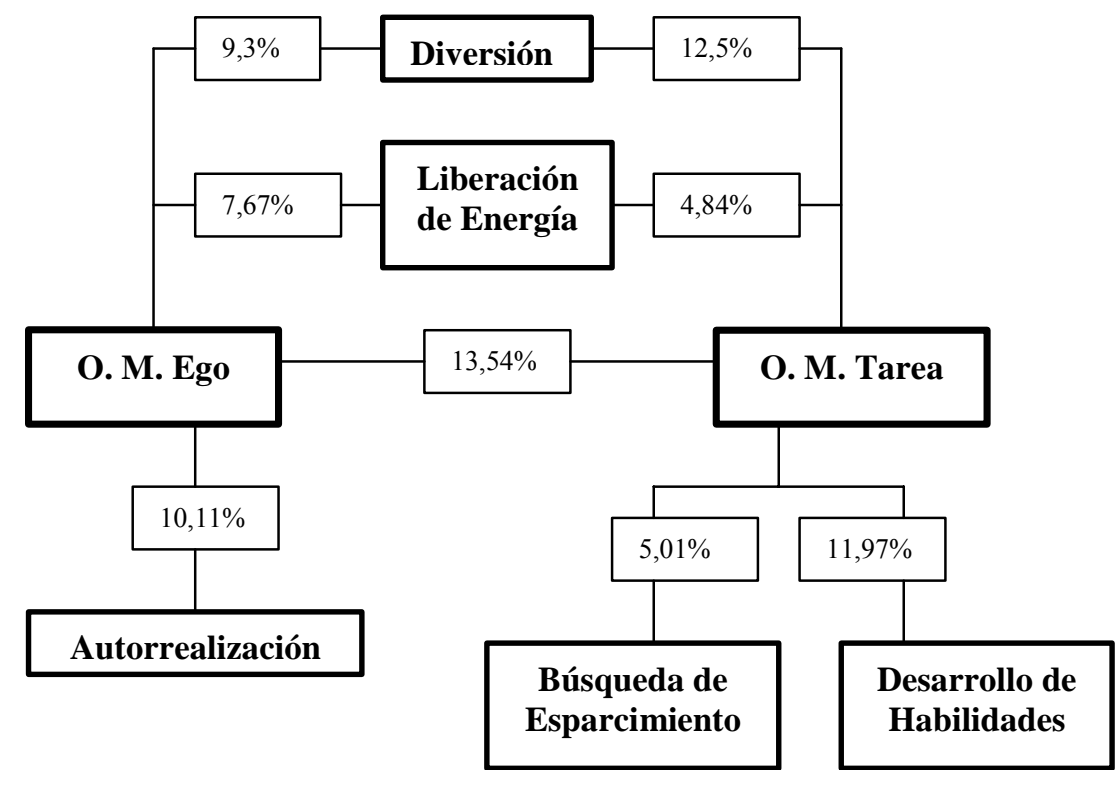

Según se aprecia en el esquema 1, el porcentaje de varianza explicada de un $13,54 \%$, hallado entre las orientaciones de metas a la tarea y al ego en hombres indica que ambas están vinculadas entre sí, en estos sujetos. Así mismo, los motivos relacionados con el factor motivacional "diversión", se ven explicados tanto por la orientación al ego como a la tarea y lo mismo ocurre con los motivos relacionados con el factor "liberación de energía". Los motivos de "autorrealización" se explican solo por la orientación al ego, mientras que los motivos de "búsqueda de esparcimiento" y "desarrollo de habilidades" solo se explican por la orientación a la tarea. Por tanto, la teoría de orientación de metas sirve parcialmente, para comprender la naturaleza de los motivos de participación deportiva de los hombres 
entrevistados, pero no sirvió para este efecto, para el caso de las deportistas analizadas.

\section{DISCUSIÓN}

Los resultados señalan que no se encontraron diferencias significativas en la preferencia de los hombres y mujeres hacia determinados motivos de participación deportiva, lo que indica que el patrón fue muy similar entre deportistas de ambos sexos a lo largo de todo el rango de edad de la presente muestra (8-23 años). Estos resultados van en contra de lo expresado en parte de la literatura previa en este campo, la que indica que los hombres participan por estatus social, por la competición y la liberación de energía (Gould y cols., 1985; Rosich, 2005; Weinberg y cols. 2000; Longhurst y Spink, 1987; Sapp y Haubenstricker; 1978), mientras las mujeres suelen tener metas de control de peso y estética y han demostrado dar énfasis al aspecto social, dando mayor importancia a la relación de pares, a la amistad, la diversión, el trabajo en equipo y a la condición física (Petherick y Weigand, 2002; Rosich, 2005; Isogai y cols, 2003; Weinberg y cols. 2000; Longhurst y Spink, 1987; Gould y cols. 1985). Por otro lado, los resultados apoyan lo reportado por Alderman (1978); Gill y cols (1983); Balaguer y Atienza (1994) y González y cols (2000), quienes tampoco encontraron diferencias significativas en función del sexo, indicando que, hombres y mujeres, coinciden en los motivos principales para participar en deporte.

La importancia que tienen las dos orientaciones de metas es muy similar entre los y las deportistas estudiados, ya que tampoco existieron diferencias ligadas al sexo. Esto es diferente a lo encontrado en estudios previos (Zúñiga, 2005; White, Kavussanu y Guest, 1998; Balaguer, Castillo y Tomás, 1996; White y Duda, 1994; Battista, 1990; Duda, 1989; Gill y cols 1996). Según esas investigaciones, las mujeres suelen ser más orientadas a la tarea que los hombres y estos, se orientan más al ego que las mujeres. Sin embargo, los resultados fueron similares a lo encontrado por varios autores que no han podido confirmar las diferencias en la orientación de metas entre los sexos (Duda y Horn, 1993; White y Zellner, 1996; Etnier, Sindman y Hancock, 2004; White y Duda, 1994).

En cuanto a la edad, la participación deportiva llega a un nivel máximo en torno a los doce años (Estado de Michigan, 1976). Gould y Horn (1984), indicaron que la mayoría de los niños de etapa escolar y posterior a ella, tuvieron respuestas similares entre hombres y mujeres, sobre las razones para practicar deporte, siendo principalmente por divertirse, hacer algo para lo que estaban dotados, mejorar las destrezas, entre otros; así mismo, en cuanto a los jóvenes, para Gould y Petlichkoff (1988) las razones para participar son el aprendizaje de nuevas destrezas, divertirse, afiliación, entusiasmo y emociones, ejercicio y forma física y desafíos competitivos/victoria. Según los resultados obtenidos, existen diferencias en cuanto a la importancia que le dan los deportistas de diferentes edades, a algunos factores motivacionales; los motivos referentes a la diversión, la búsqueda de esparcimiento y la forma física, fueron más importantes conforme se avanzaba en edad. Así mismo, el factor forma física, fue el más importante en los tres grupos de edad estudiados, no obstante, en el caso de los sujetos de 12 años o menos, este factor comparte su importancia con el desarrollo de habilidades y los factores sociales, y en los sujetos de 13 a 19 años, forma física comparte importancia solo con desarrollo de habilidades; en los sujetos de 20 años o más, la forma física es claramente el factor motivacional más importante; la diversión y la búsqueda de esparcimiento se destacaron más de los otros factores motivacionales a partir de los 13 años, pero compartiendo importancia con el desarrollo de habilidades. Por lo que resulta interesante centrarse en distintos grupos de edad y analizar con profundidad sus preferencias.

Los estudios previos en esta área, han relacionado positiva y significativamente diferentes motivos para la práctica deportiva con la orientación de metas a la tarea; por ejemplo, los factores habilidades/competición y atmósfera de 
equipo (Zahariadis y Biddle, 2000); los factores desarrollo de habilidades y disfrute (Papaioannou y Theodorakis, 1994); factores afiliación y salud (Castillo, Balaguer y Duda, 2000) y finalmente, los factores desarrollo de habilidades, forma física, afiliación, formar parte de un equipo y competición (White y Duda, 1994).

En el presente estudio, en el caso de los hombres, los factores "diversión, liberación de energía, desarrollo de habilidades y búsqueda de esparcimiento", correlacionaron positivamente con la orientación de metas a la tarea, lo que indica que a mayor importancia a estos motivos de participación deportiva, más se orienta el deportista a la tarea. De estos resultados se aprecia la coincidencia del factor desarrollo de habilidades con lo encontrado por Papaioannou y Theodorakis (1994), White y Duda (1994) y por Zahariadis y Biddle (2000).

de $\begin{array}{llr}\text { En cuanto a los factores de motivos } \\ \text { participación deportiva que }\end{array}$ correlacionaron positivamente con la orientación de metas al ego, Zahariadis y Biddle (2000), Papanoionnou y Theodorakis (1994) y White y Duda (1994), indicaron congruentemente los factores referentes a la obtención de estatus y reconocimiento, mientras Castillo, Balaguer y Duda (2000), indicaron los motivos aprobación social/demostración de capacidades. En el presente estudio, los factores "autorrealización, diversión y liberación de energía", correlacionaron positivamente con la orientación al ego, coincidiendo en cuanto al factor autorrealización con los resultados de Zahariadis y Biddle (2000), Papanoionnou y Theodorakis (1994) y White y Duda (1994).

De los estudios anteriormente citados, solamente el estudio de Castillo y cols (2000) analizó la diferencia entre sexos en cuanto a la relación entre los motivos de participación deportiva y la orientación de metas. Los resultados mostraron para los hombres, que los factores: diversión, liberación de energía, búsqueda de esparcimiento y desarrollo de habilidades correlacionaron positivamente con la orientación de metas a la tarea, indicando la tendencia de que a mayor importancia tengan tales factores, mayor será la orientación a la tarea. Ninguno de estos coincide con los factores de motivos de participación deportiva indicados por Castillo y cols (2000), en que los factores afiliación y salud, fueron los que correlacionaron positivamente con la orientación de metas a la tarea entre los hombres. Para las mujeres, en el presente análisis, ningún factor se relacionó significativamente con las orientaciones a la tarea, hecho que difiere de lo hallado por Castillo y cols (2000), quienes encontraron en las mujeres relación con los motivos de afiliación. El cuestionario "The Health Behavior School-Children" traducido al castellano (Balaguer, 1999) y utilizado por Castillo y cols. (2000) hace referencia a motivos más ligados a la salud. Además, en ese estudio, los sujetos eran participantes de lecciones de educación física y en el presente estudio eran deportistas, los cuales son sujetos para quienes tales motivos pueden tener menor relevancia.

En cuanto a la orientación de metas a la tarea, en los hombres se encontró mayor relación entre motivos de participación y esta orientación. Por tanto, los resultados no van de acuerdo con lo esperado, que indicaba que en las mujeres se encontraría mayor relación entre factores motivacionales con la orientación de metas a la tarea, pues varios estudios señalaron la preferencia de las mujeres por dicha orientación (Zúñiga, 2005; White, Kavussanu y Guest, 1998; Balaguer, Castillo y Tomás, 1996).

Respecto a la orientación de metas al ego, en el estudio realizado por Castillo y cols (2000), tanto en hombres como en mujeres, esta orientación se relacionó significativamente con el factor "aprobación social/demostración de capacidades", que incluía motivos como "ser una estrella, ser bueno, ganar y agradar a los padres". En el presente estudio, tanto para hombres como para las mujeres, el factor “autorrealización” correlacionó positivamente con la orientación al ego; lo que indica que a mayor importancia se da a este factor, mayor orientación al ego. El factor "autorrealización" incluye motivos referentes a obtener prestigio y 
reconocimiento, a ganar, sentirse importante, ser famoso y ganar premios, por lo que es evidente la similitud con lo encontrado por Castillo y cols. Además, para la muestra de hombres, dos factores más, diversión $y$ liberación de energía, correlacionaron positivamente con la orientación al ego y también con la orientación a la tarea; no obstante, ninguno de estos últimos factores se contempló en el modelo estudiado por Castillo y cols. (2000).

Ambos sexos coinciden en cuanto a la correlación del factor autorrealización con la orientación al ego y en cuanto a la ausencia de correlación significativa de los factores "trabajo en equipo, factores sociales y forma física" con ninguna de las orientaciones de metas, lo que representa una tendencia general en los sujetos participantes de este estudio.

En conclusión, según el sexo, no se encontraron diferencias en la importancia que le dan hombres y mujeres a los motivos de participación deportiva y a las orientaciones de metas a la tarea y al ego, sin embargo, se encontró, que para ciertos grupos de edad, algunos motivos son más importantes a la hora de explicar su participación en deporte y también se encontró diferencias en la orientación de metas a la tarea, según la edad.

Existen diferencias en cuanto a la relación que existe entre motivos de participación deportiva y la orientación de metas entre hombres y mujeres, ya que, en los hombres, tres motivos de participación deportiva (Diversión, Liberación de Energía y Autorrealización) podrían explicar su orientación al ego (o viceversa); así mismo, 4 motivos de participación (Diversión, Liberación de Energía, Búsqueda de esparcimiento y Desarrollo de habilidades) sirven para explicar su orientación de metas a la tarea o viceversa; y además dos motivos de participación (Diversión y Liberación de energía) podrían explicarse tanto con base en su orientación de metas al ego, como con su orientación a la tarea. En las mujeres el comportamiento es diferente, ya que solo un motivo (Autorrealización) podría explicarse por la orientación motivacional al ego y ninguno se explica por la orientación a la tarea ni por ambas orientaciones.

Por tanto, la teoría de orientación de metas resultó ser útil para explicar algunos motivos de participación deportiva de los hombres, pero no tuvo la misma utilidad para explicar los motivos de participación de las mujeres. Futuros estudios deberían aplicar modelos teóricos más integrales (mayor número de variables), que faciliten entender mejor el fenómeno de la motivación para la participación deportiva, especialmente en el caso de las mujeres.

La discusión sobre la teoría de orientación de metas en muchos casos (Duda y White, 1992; Treasure y Roberts, 1994) ha caído en un extremismo en que se asume que se está orientado a la tarea o al ego como dos variables opuestas, diferentes e independientes, sin embargo, el ser humano es muy complejo y es probable que la orientación de metas en algunos aspectos pueda recaer en combinaciones de ambas orientaciones, o que ambas vayan en la misma dirección, lo que se puede probar en futuros estudios buscando sub-orientaciones que se podrían presentar en función de la disciplina practicada o las características de la población.

Futuros estudios podrían también ayudar a responder la incertidumbre de si la orientación de metas es un rasgo y si es así, con qué características de la personalidad se relaciona. Finalmente, se podría investigar qué tanto influye la orientación de metas en la conducta más allá del ambiente deportivo (por ejemplo la toma de decisiones de un sujeto que practica actividad física por salud) y qué tanto la orientación de metas podría incidir en su adherencia a este tipo de programas.

\section{REFERENCIAS}

Alderman, R. B. (1978). Strategies for motivating young athletes. En W. F. Straub (Ed.) Sport psychology: An analysis of athlete behaviour. Ithaca, New York. Mouvement Publications.

Alderman, R. B., y Wood, N. L. (1976). An analysis of incentive motivation in young Canadian athletes. Canadian Journal of Applied Sports Science, 1, 169-176. 
Ames, C. (1992). Achievement goals, motivational climate and motivational processes. En G. C. Roberts (Ed.), Motivational in sport and exercise. Champaign, IL: Human Kinetics. Pp. 161-176. En Cervelló, E.M. y Santos-Rosa, F.J. (2001). Motivation in sport: An achievement goal perspective in young spanish recreational athletes. Perceptual and Motor Skills. 92, 527-534.

Ames, C., y Archer, J. (1988). Achievement goals in the classroom: students' learning strategies and motivation process. Journal of Educational Psychology. 80, 260-267.

Araya, G.A. (2000). La violencia en el deporte: Estudio retrospectivo sobre la relación entre la conducta agresiva, orientación de metas y el razonamiento moral en futbolistas. Tesis de Maestría en Ciencias del Movimiento Humano. Universidad de Costa Rica. Costa Rica.

Balaguer, I. y Atienza, F. (1994). Principales motivos de los jóvenes para jugar al tenis. Apunts: Educación Física y Deportes, 31, 285-299.

Balaguer, I. (1999). Estilo de vida de los adolescentes de la Comunidad Valenciana: Un estudio de la socialización para estilos de vida saludable. En: Castillo, I. Balaguer, I y Duda, J.L. (2000). Las Orientaciones de meta y los motivos de práctica deportiva en los jóvenes deportistas valencianos escolarizados. Revista de Psicología del Deporte. 9 (1-2). 37-50.

Balaguer, I., Castillo, I., y Tomás, I. (1996). Análisis de las propiedades psicométricas del cuestionario de orientación al ego y a la tarea en el deporte (TEOSQ) en su traducción al castellano. Psicológica, 17, 71-81.

Battista, R.R. (1990). Personal meaning: Attraction to sports participation. Perceptual and Motor Skills, 70, 1003-1009.

Bergerone, C., Cei, A. y Ceridono, D. (1985). Motivations in the trainer-child relationship. International Journal of Sport Psychology. 16: 150-157.

Blasco, T., Capdevilla, Ll., Pintanel, M., Valiente, L. y Cruz, J. (1996). Evolución de los patrones de actividad física en estudiantes universitarios. Revista de Psicología del deporte, 9-10, 51-63.

Cale, L. (1996). An assessment of the physical activity levels of adolescent girls - implications for Physical Education. European Journal of Physical Education, 1, 46-55.

Campbell, P., MacAuley, D., McCrum, E. y Evans, A. (2001) Age Differences in the motivational factors for exercise. Journal of Sport and Exercise Psychology, 23, 191-199.

Castillo, I. Balaguer, I y Duda, J.L. (2000). Las Orientaciones de meta y los motivos de práctica deportiva en los jóvenes deportistas valencianos escolarizados. Revista de Psicología del Deporte. 9 (1-2). 37-50.

Castillo, I. ; Balaguer, I. y Duda, J.L. (2002). La perspectiva de meta de los adolescentes en el contexto deportivo. Psicothema. 14 (2), 280-287.

Cervelló, E.M. y Santos-Rosa, F.J. (2001). Motivation in sport: An achievement goal perspective in young spanish recreational athletes. Perceptual and Motor Skills. $92,527-534$.

Cookburn, C. (2000). Las opiniones de chicas de 13 y 14 años sobre la Educación Física en las escuelas públicas británicas. Estudio realizado en los condados de Hampshire y Cambridgeshire. Apunts: Educación Física y Deportes, 62, 91-101.

Duda, J.L. (1989). The relationship between task and ego orientation and the perceived purpose of sport among high school athletes. Journal of sport and exercise psychology, 11, 318-335.

Duda, J.L. (1993). Goals: A social cognitive approach to the study of achievement motivation in sport. En Singer, R.N., Murphey, M., y Tennant (Eds.), Handbook on research in sport psychology (pp. 421-436). New York: Macmillan.

Duda, J.L., Chi, L., Newton, M.L., Walling, M.D., Catley, D. (1995). Task and ego orientation and intrinsic motivation in sport. International journal of sport psychology. 26 (1). p. 40-63.

Duda, J.L., Fox, K.R., Biddle, S.J.H., y Armstrong, N. (1992). Children's achievement goals and beliefs about success in sport. British Journal of Educational Psychology, 62, 309-319.

Duda, J.L., y Horn, H.L. (1993). Interdependencies between the perceived and self-reported goal orientations of young athletes and their parents. Pediatric Exercise Science, 5, 234-241.

Duda, J.L., y White, S.A. (1992). The relationship of goal perspectivas to beliefs about success among elite skiers. The Sport Psychologist, 6, 334-343.

Durand, M. (1988). El niño y el deporte. $2^{\circ}$ Edición. Barcelona, España.Ediciones Paidos.

Dweck, C.S. (1986). Motivational processes affecting learning. American Psychologist, 41, 1040-1048.

Dweck, C.S., y Elliot, E.S. (1983). Achievement motivation. En Hetherington (Ed.), Handbook of child psychology: Vol 4: Socialization, personality and social development (pp. 643-691). New York: Wiley.

Dweck, C.S. y Leggett, E.L. (1988). A social cognitive approach to motivation and personality. Psychological review, 95, 1-18.

Estado de Michigan (1976). Joint legislative study on youth sports programs. Phase 2, East Lansing, MI, Autor. En Weinberg, R.S. y Gould, D. (1996). Fundamentos de psicología del deporte y el ejercicio físico. Barcelona, España. Editorial Ariel, S.A.

Etnier, J. L., Sidman, C. L. y Hancock, L. C. (2004). An examination of goal orientation profiles and motivation in adult team sport. International Journal of Sport Psychology. 35: 173-188.

Gill, D.L., Gross, J.B. y Huddleston, S. (1983). Participation motivation in youth sports. International Journal of Sport Psychology. 14: 1-14.

González, G. Tabernero, B. y Márquez, S. (2000). Análisis de los motivos para participar en fútbol y en tennis en la iniciación deportiva. Revista motricidad. 6: 4766.

Gould, D. y Horn, T. (1984). Participation motivation in young athletes. En Silva y Weinberg (eds.), Psychological foundations of sport (pp. 359-370), Champaign, IL, Human Kinetics.

Gould, D. y Petlichkoff, L. (1988). Participation motivation and attrition in young athletes. En Smoll, Magil y Ash (eds.), Children in sport (3ra ed., pp. 161-178), Champaign, IL, Human Kinetics.

Gould, D; Feltz, D; y Weiss, M. (1985). Motives for participating in competitive youth swimming. International Journal of Sport Psychology. 16. 126140.

Guivernau, M. y Duda, J. (1994). Psychometric properties of a spanish version of the task and ego orientation in sport questionnaire (TEOSQ) and beliefs about the causes of success inventory. Revista de psicología del deporte, 5, 31-51.

Hellandsig, E. T. (1998). Motivational predictors of high performance and discontinuation in different types 
of sports among talented teenage athletes. International Journal of Sport Psychology, 29, 2744.

Isogai, H., Brewer, B. W., Cornelius, A. E., Etnier, J. y Tokunaga, M. (2003). International Journal of Sport Psychology. 34: 80-93.

Kim, M.S., Chang, D.S. y Gu, H. (2003). Difference in skill levels and gender in achievement goals, perceptions of motivational atmosphere, and motivation in sport. Journal of Sport and Exercise Psychology. 25: supplement. p. S81.

Longhurst, K., y Spink, K.S. (1987). Participation motivation of Australian children involved in organized sport. Canadian Journal of applied Sport Sciences, 12, 24-30.

Lorenzo, J. (1997). Psicología del Deporte. Madrid, España. Editorial Biblioteca Nueva.

Martínez, R., Andrade, A. y Salguero, A. (2005). Análisis factorial del cuestionario de motivos de participación deportiva de Gill y cols. (1983) Adaptado a una muestra de jóvenes futbolistas. Memoria del $X$ Congreso Nacional y Andaluz de Psicología de la Actividad Física y el Deporte. Málaga, España. 291-302.

Maehr, M.L. y Nicholls, J.G. (1980). Culture and achievement motivation: A second look. En N. Warren (Ed), Studies in cross-cultural psychology (Vol 2, pp. 221-267). New York: Academic Press.

Nicholls, J.G. (1984). Achievement motivation: Conceptions of hability, subjective experience, task choice, and performance. Psychological review, 91, 328-346.

Nicholls, J. G. (1989). The competitive ethos and democratic education. Cambridge. MA: Harward University Press.

Nigg, C. R. (2003). Do sport participation motivations add to the ability of the transtheoretical model to explain adolescent exercise behavior? International Journal of Sport Psychology, 34, 208-225.

Papaioannou, A. \& Theodorakis, Y. (1994). Attitudes values and goals: A test of three models for the prediction of intention for participation in physical education. En Zahariadis, P.N., y Biddle, S.J. (2000). Goal orientations and participation motives in physical education and sport: their relationship in english schoolchildren. Journal of sport Psychology.

Pavón, A. I., Moreno, J. A., Gutiérrez, M. y Sicilia, Á. (2003). Interés y motivaciones de los universitarios: diferencias en función del nivel de práctica. Cuadernos de Psicología del Deporte, 3(1), 33-43.

Petherick, C. M., y Weigand, D. A. (2002). The relationship of dispositional goal orientations and perceived motivational climates on indices of motivation in male and female swimmers. International Journal of Sport Psychology. 33: 218-237.

Roberts, G.C. (1984). Achievement notivation in children's sport. En Nicholls, J. (Ed.), The development of achievement motivation (pp. 251-281). Greenwich, CT: JAI Press.

Roberts, G.C. y Treasure, D.C. (1995). Achievement goals, motivational climate and achievement strategies and behaviors in sport. International Journal of sport psychology. 26, 64-80.

Rosich, M. (2005). Estudio sobre la percepción de satisfacción en el deporte en el ámbito competitivo en una muestra de universitarios. Memoria del X Congreso Nacional y Andaluz de Psicología de la Actividad Física y el Deporte. Málaga, España. 632-641.

Saap. M. y Haubenstricker, J. (1978). Motivation for joining and reasons for not continuing in youth sports programs in Michigan. Paper presented at
AAHPER Conference, Kansas City, MO. En Balaguer, I. y Atienza, F. (1994). Principales motivos de los jóvenes para jugar al tenis. Appunts: Educación Física y Deportes, 31, 285-299.

Seifriz, J.J., Duda, J.L. y Chi, L. (1992). The relatioship of perceived motivaional climate to intrinsic motivations and beliefs about success in basketball. Journal of Sport and Exercise Psychology. 14: 375391.

Simons, J., Dewitte, S. y Lens, W. (2003). "Don't do it for me. Do it for yourself!" Stressing the personal relevance enhances motivation in physical education. Journal of Sport and Exercise Psychology. 25: 145-160.

Smith, C. y Storandt, M. (1997). Physical Activity Participation in Older Adults: A comparison of Competitors, Noncompetitors and Nonexersice. Journal of Aging and Physical Activity, 5, 98-110.

Tabernero, B. (1998). Motivos para practicar tenis en la iniciación deportiva. Ponencia presentada en memoria del II Congreso Internacional sobre la Enseñanza de la Educación física y el Deporte Escolar, Málaga, Instituto Andaluz del deporte, España.

Treasure, D. C. y Roberts, G. C. (1994). Cognitive and affective concomitants of task and ego goal orientation during the middle school years. International Journal of Sport and Exercise Psychology. 16, 15-28.

Valdés, H. M. (1996). La preparación psicológica del deportista. Mente y rendimiento humano. I edición, España. INDE publicaciones. p.54-57.

Villegas, C.A. (2003). Relación entre la motivación intrínseca para la práctica del fútbol y la orientación subjetiva de los padres y entrenadores hacia el resultado o la tarea percibida por niños de 10 a 13 años. Tesis de Licenciatura en Ciencias del Deporte con énfasis en Rendimiento Deportivo. Escuela Ciencias del Deporte, Universidad Nacional. Heredia, Costa Rica.

Vlachopoulos, S., Biddle, S. y Fox, K. (1996). A socialcognitive investigation into the mechanisms of affect generation in children's physical activity. Journal of Sport and Exercise Psychology. 18: 174193.

Wang, J. y Weise-Bjornstal, D. M. (1996). The relationship of school type and gender to motives for sport participation among youth in the people's Republic of China. International Journal of Sport Psychology, 28, 13-24.

Weigand, D.A. y Broadhurst, C.J. (1998). The relationship among perceived competence, intrinsic motivation, and control perceptions in youth soccer. International Journal of Sport Psychology, 29, 324338.

Weinberg, R.S. y Gould, D. (1996). Fundamentos de psicología del deporte y el ejercicio físico. Barcelona, España. Editorial Ariel, S.A. p. 230-250.

Weinberg, R., Tenenbaum, G., McKenzie, A., Jackson, S., Ashel, M., Grove, R. y Fogarty, G. (2000). Motivation for youth participation in sport and physical activity: relationships to culture, selfreported activity levels, and gender. International Journal of Sport Psychology. 31: 321-346.

White, S.A., y Duda, J.L. (1994). The relationship of gender, level of sport involvement, and participation motivation to task and ego orientation. International Journal of Sport Psychology. 26, 418.

White, S.A., Kavussanu, M., y Guest, S.M. (1998). Goal orientation and perceptions of the motivational 
climate created by significant others. Manuscript under review. En Petherick, C. M., y Weigand, D.

A. (2002). The relationship of dispositional goal orientations and perceived motivational climates on indices of motivation in male and female swimmers. International Journal of Sport Psychology. 33: 218-237.

White, S.A., y Zellner, S.R. (1996). The relationship between goal orientation, beliefs about causes of sport success, and trait anxiety among high school, intercollegiate, and recreational sports participants. The Sport Psychologist. 10, 58-72.

Williams, L. y Cox, A. (2003). The relationship among social motivational orientations, perceived social belonging, and motivation-related outcomes. Journal of Sport and Exercise Psychology. 25: supplement. p. S7-S8.

Zahariadis, P.N., y Biddle, S.J. (2000). Goal orientations and participation motives in physical education and sport: their relationship in english schoolchildren. Journal of Sport Psychology.

Zúñiga, K. (2005). Fuentes de motivación para la práctica de actividades acuáticas (natación y aeróbicos) en mujeres mayores de treinta años de edad de diversas nacionalidades. Tesis de Licenciatura en Ciencias del Deporte con énfasis en Rendimiento Deportivo. Escuela Ciencias del Deporte, Universidad Nacional. Heredia, Costa Rica. 